\title{
Determination of the color changes against accelerated UV aging of used water based layers on some heat-treated (ThermoWood) wood species
}

\author{
Umit AYATA ${ }^{1}$, Nevzat CAKİCİER ${ }^{2 *}$
}

\begin{abstract}
${ }^{1}$ Ataturk University, Oltu Vocation School, Forestry and Forest Products, Oltu/Erzurum, Turkey, ${ }^{2 *}$ Duzce University, Faculty of Forestry, Department of Forest Industrial Engineering, Duzce, Turkey. *Corresponding author: nevzatcakicier@duzce.edu.tr
\end{abstract}

Received Date: 19.04.2017

Accepted Date: 17.11.2017

\section{Abstract}

Aim of study: The objective of this study is to investigate the accelerated UV resistance of water-based varnish layers, applied on heat-treated wood (ThermoWood) surfaces.

Area of study: Determination of the relationship between heat-treated, water based varnish, aging and color.

Material and Methods: Wood specimens prepared from Scotch pine (Pinus sylvestris L.), Oak (Quercus petreae L.) and Oriental beech (Fagus orientalis L.) were heat-treated according to ThermoWood method at $190^{\circ} \mathrm{C}$ for 2 hours, and at $212^{\circ} \mathrm{C}$ for 1 hour and 2 hours. Following the heat treatment, one and two component water-based varnishes were applied to similar layer thicknesses. The finished specimens were exposed to UV-A $340 \mathrm{~nm}$ fluorescent lamp in a "QUV accelerated weathering tester” for 144, 288 and 432 hours (ASTM G 154-06, 2006). At the end of each exposure period, red color tone, yellow color tone, lightness and total color difference values were determined according to ASTMD 2244-3.

Main results: Lightness and total color difference values of the water-based single and double component varnishes increased after the UV aging.

Research highlights: All color parameters had changed due to aging.

Keywords: Heat Treatment, UV Aging, Water-Based Varnish, Color

Isıl işlem (ThermoWood) görmüş bazı odun türlerinde uygulanan su

bazlı vernikler katmanlarının UV yaşlandırma etkisine karşı renk

\section{değişikliklerinin belirlenmesi}

\section{Özet}

Çalışmanın amacı: Isıl işlem (ThermoWood) görmüş bazı ağaç türlerinde kullanılan su bazlı vernik katmanlarının hızlandırılmış UV yaşlandırma etkisine karşı renk değişim özelliklerini belirlemektir.

Çalışma alanı: Isıl işlem görmüş, su bazlı vernik, yaşlandırma ve renk arasındaki ilişkinin belirlenmesidir.

Materyal ve Yöntem: Bu amaç ile Sarıçam (Pinus sylvestris L.), Sapsız meşe (Quercus petreae L.) ve Doğu kayını (Fagus orientalis L.) odunlarından hazırlanan deney örnekleri ThermoWood metoduna göre $190^{\circ} \mathrm{C}$ 'de 2 saat, $212^{\circ} \mathrm{C}^{\prime}$ de 1 saat ve 2 saat süreler ile isıl işleme tabi tutulmuştur. Daha sonra deney örneklerinin yüzeylerine, tek ve çift bileşenli su bazlı vernikler üretici firmaların önerilerine göre uygulanmış ve UV-A 340 nm florasan lambalarının bulunduğu "QUV accelerated weathering tester" cihazında; 144, 288 ve 432 saat süre boyunca UV etkisine altında (ASTM G 154-06, 2006) yaşlandırmaya maruz bırakılmışlardır. Yaşlandırma sonrasında, kırmızı renk tonu, sarı renk tonu, işıklılık ve toplam renk farkı değerleri ASTM-D 2244-3 standardına göre belirlenmiş̧tir.

Sonuçlar: Yaşlandırma sonrasında ışıklılık ve toplam renk farkı değerlerinin artış gösterdiği belirlenmiştir.

Araşstrma vurguları: Yaşlandırmaya bağlı olarak bütün renk parametreleri değişmiştir.

Anahtar kelimeler: Isıl işlem, UV yaşlandırma, Su-bazlı vernik, Renk 


\section{Introduction}

Wood is a natural composite material. The protection of wooden materials is necessary. There are many studies on this subject (Kurtoglu, 2000; Bozkurt, Goker \& Erdin 1993; Korkut \& Kocaefe, 2009; Ayata, 2014). There are many different methods in the literature (impregnation, acetylation, etc.) for the protection of wood materials. These protection methods generally contain chemical substances. A sample of this method, Sogutlu \& Sonmez (2006) were reported that discoloration performances of acacia, pear, chestnut, oak and cedar applied shellac varnish, teak oil, and liquid paraffin wax after exposure of UV lights for 72 hours. One of these methods is the heat treatment method. But, temperature and water vapor are used in this method. Today, there are various heat treatment methods. ThermoWood method is one of these methods and is located in our country (Bolu-Gerede, Turkey). Heattreated material is used in outdoor environment. But heat-treated wood material is exposed to external influences (wind, rain, frost, temperature, etc.). For this, surface protective chemicals (varnish, paint, etc.) can be applied to heat-treated wood materials. The color change in wooden materials has due to various reasons. As an example of this work: it was stated that there was a strong correlation between the value of color lightness for hemicellulose in Oriental beech wood, lignin in pine wood and gluxy in a spruce wood (GonzalezPena \& Hale, 2009). Considering total color difference results, it can be seen that these values changed. In another study, the color change in wood after heat treatment was due to the hydrolysis of hemicelluloses (Hillis, 1975). Akkus (2012) reported variation in $\Delta E^{*}$ could be a chemical change that can occur in the main polymers of heat-treated wood. In the case of scots pine wood, it was reported that extractives were more abundant than other species and that oxidation-ending color changed too much with water-based varnishes exhibiting alkali (pH 8-9) (Cakicier, 2007). Another study reported that high energy of sunlight ultraviolet (UV) wave lengths causes deterioration of varnish and paint layers (Feist, 1984).

In this study, color parameters against the aging effect of heat-treated Scotch pine, Oak and Oriental beech wood species (at $190^{\circ} \mathrm{C}$ for 2 hours, $212^{\circ} \mathrm{C}$ for 1 hour to 2 hours according to ThermoWood method) and water based varnishes (single and double component) applied samples were investigated. These results will help determine the relationship between aging - heat treatment - varnish.

\section{Material and Method Wood Materials}

In this study, Scots pine (Pinus sylvestris L.), Oriental beech (Fagus orientalis L.) and oak (Quercus petreae L.) were chosen. These wood species were chosen because they are widely used species in the furniture and decoration industry in our country (Kazan, 2009). These wood types were taken from factories in Duzce, Turkey by measuring dimensions $510 \times 110 \times 20$ $\mathrm{mm}$ fresh according to random selection method. Test specimens were chosen to be equally radial and tangential. Wood samples were kept at a temperature of $20 \pm 2^{\circ} \mathrm{C}$ and a humidity of $65 \pm 5 \%$ relative humidity until reaching a constant weight, resulting in a moisture uptake of 12\% (TS 642, 1997).

\section{Heat Treatment Application}

Wood samples were subjected to heat treatment at $190^{\circ} \mathrm{C}$ for 2 hours and at $212^{\circ} \mathrm{C}$ for 1 hour and 2 hours according to ThermoWood method (Nova ThermoWood Factory, Bolu-Gerede, 
Turkey) (Anonymous, 2003). Later, test specimens were sanded 100, 120 and 180 sand in the calibrated sanding machine according to industrial applications. The test specimens were determined as $500 \mathrm{x}$ $100 \times 14 \mathrm{~mm}$. Heat-treated samples were kept until they reached constant weight at an average temperature of $20 \pm 2^{\circ} \mathrm{C}$ and a humidity of $65 \pm 5 \%$ relative humidity (TS 642 1997).

\section{Application of Varnishes Application of Primer Varnish}

Heat-treated lumbers

were impregnated as a dip method in a 10 second period according to the company's recommendations. This process was applied twice. AQUACOOL FX 6150 was supplied by DUAL Boya Firm. It has a colorless liner containing coded biocide and lignin preservative. After, waiting for 3 hours at $20^{\circ} \mathrm{C}$ ambient temperature, the dried varnish film 400 was sanded with water and applied on the second layer after the dusts were cleaned. Later thoroughly dry sanding with sanding pad number 400 and cleaning the dusts, water based single and double component varnish application was performed.

\section{Application of Water Based Single and Double Component Varnishes}

In this study, water based single component varnish (AQUACOOL FX $7680 / 00$ ) and water based double component varnish (AQUACOOL 0820/00) were used. Water based double component varnish application was applied to varnish + AQUACOOL AX 0115 hardener (25\%) + water (10\%).

Table 1. Application of water-based single and double component varnishes

\begin{tabular}{|c|c|c|c|c|}
\hline \multirow{5}{*}{$\begin{array}{l}\text { Water-based single } \\
\text { component varnish } \\
\text { application }\end{array}$} & \multirow{2}{*}{$\begin{array}{l}\text { FX } 6150 \text { UV protective primer immersion method } \\
\text { (Solid matter } 19.45 \% \text { ) }\end{array}$} & 1. layer & $130 \mathrm{~g} / \mathrm{m}^{2}$ & $25 \mathrm{~g} / \mathrm{m}^{2}$ \\
\hline & & 2. layers & $70 \mathrm{~g} / \mathrm{m}^{2}$ & $13 \mathrm{~g} / \mathrm{m}^{2}$ \\
\hline & \multirow{2}{*}{$\begin{array}{l}\text { FX } 7680 \text { finish coating method with pistole } \\
\text { (Solid matter 43.26\%) }\end{array}$} & 1. layer & $140 \mathrm{~g} / \mathrm{m}^{2}$ & $61 \mathrm{~g} / \mathrm{m}^{2}$ \\
\hline & & 2. layers & $140 \mathrm{~g} / \mathrm{m}^{2}$ & $61 \mathrm{~g} / \mathrm{m}^{2}$ \\
\hline & Total solids & & & $160 \mathrm{~g} / \mathrm{m}^{2}$ \\
\hline \multirow{6}{*}{$\begin{array}{l}\text { Water-based double } \\
\text { component varnish } \\
\text { application }\end{array}$} & \multirow{2}{*}{$\begin{array}{l}\text { FX } 6150 \text { UV protective primer immersion method } \\
\text { (Solid matter } 19.45 \%)\end{array}$} & 1. layer & $130 \mathrm{~g} / \mathrm{m}^{2}$ & $25 \mathrm{~g} / \mathrm{m}^{2}$ \\
\hline & & 2. layers & $70 \mathrm{~g} / \mathrm{m}^{2}$ & $13 \mathrm{~g} / \mathrm{m}^{2}$ \\
\hline & \multirow{3}{*}{$\begin{array}{l}\text { FX } 08202 \mathrm{~K} \text { finish coat method with piston pistole } \\
\text { (Solids } 37.78 \% \text { ) }\end{array}$} & 1. layer & $105 \mathrm{~g} / \mathrm{m}^{2}$ & $40 \mathrm{~g} / \mathrm{m}^{2}$ \\
\hline & & 2. layers & $105 \mathrm{~g} / \mathrm{m}^{2}$ & $40 \mathrm{~g} / \mathrm{m}^{2}$ \\
\hline & & 3. layers & $105 \mathrm{~g} / \mathrm{m}^{2}$ & $40 \mathrm{~g} / \mathrm{m}^{2}$ \\
\hline & Total solids & & & $158 \mathrm{~g} / \mathrm{m}^{2}$ \\
\hline
\end{tabular}

Table 2. Information on applied varnishes

\begin{tabular}{cccccc}
\hline Varnish type & Composition & Density & pH & Solid Matter (\%) & Viscosity \\
\hline $\begin{array}{c}\text { FX 6150 UV } \\
\text { protective primer }\end{array}$ & $\begin{array}{c}\text { Acrylic resin, biocide } \\
\text { and UV protection }\end{array}$ & 1.02 & 9.2 & $19 \pm 2$ & 11 seconds at 20 $0^{\circ} \mathrm{C}$ in DIN 4 \\
cabinet
\end{tabular}

Following the heat treatment, waterbased single and double component varnishes were applied on similar layer thicknesses according to manufacturer recommendations. In the application, a spray gun with a top end capacity of 2.0 $\mathrm{mm}$ was used. Varnish applications were applied as industrial surface application with spray gun (a distance of 20-25 cm). The applications were first made perpendicular to fiber, then parallel to fiber. The air pressure in the application was chosen as 2 bars. After the application of primer varnish, the second layer for water based single component varnish and third layer for water based 
double component varnish were applied (Figure 1).

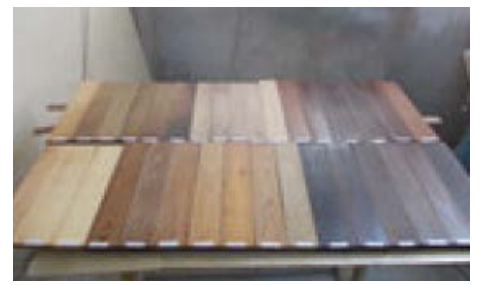

Figure 1. Application of water-based single and double component varnishes (Ayata, 2014)

\section{Application of UV Weathering Aging}

The finished specimens (120x80x14 $\mathrm{mm}$ ) were exposed to UV-A $340 \mathrm{~nm}$ fluorescent lamp in a QUV accelerated weathering tester (Figure 2) for 144, 288 and 432 hours according to ASTM G 154-06 (2006) standard. A total of 72 samples were used.

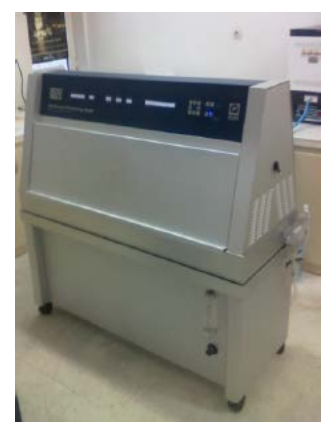

Figure 2. QUV accelerated weathering tester (Ayata, 2014)

\section{Tests}

Determination of Solid Contents

In the study, solids of water based were determined according to ASTM D 1644-01 (2006). The amounts of solids were calculated with the following formula (1, 2 and 3).

$$
\begin{aligned}
\mathrm{Km} & =[(\mathrm{Vu}-\mathrm{Cb}) / \mathrm{Vu}] \times 100 \\
\mathrm{Vu} & =\mathrm{G}-\mathrm{D} \\
\mathrm{Cb} & =\mathrm{G}-\mathrm{E}
\end{aligned}
$$

Equality;

$\mathrm{Vu}=$ Applied varnish (g)

$\mathrm{Cb}=$ Evaporating solvent $(\mathrm{g})$

$\mathrm{Km}=$ Solid matter $(\%)$

$$
\begin{aligned}
& \mathrm{G}=\text { wet weight }(\mathrm{g}) \\
& \mathrm{D}=\text { Tare }(\mathrm{g}) \\
& \mathrm{E}=\text { dry weight }(\mathrm{g})
\end{aligned}
$$

\section{Determination of Impregnated Retention Ratios}

For application of colorless primer varnish (AQUACOOL FX 6150) prepared according to the company's recommendations, a short impregnation dipping method was used and test samples were left in impregnate for 10 seconds for 2 times. Amount of solution and amount of dry matter absorbed by impregnated specimens were calculated according to following formula according to TS 5723, (1988) (Bozkurt, Goker \& Erdin, 1993).

$$
\text { Retention }=\frac{G \times C}{V} \times 10 \mathrm{~kg} / \mathrm{m}^{3}
$$

Equality;

$\mathrm{G}=$ Amount of solution absorbed by sample $\left(\mathrm{m}_{1}-\mathrm{m}_{0}\right)(\mathrm{g})$

$\mathrm{M}_{0}=$ Weight before impregnation (g)

$\mathrm{M}_{1}=$ Wet weight after impregnation (g)

$\mathrm{C}=$ Solution concentration

$\mathrm{V}=$ Volume of wood sample $\left(\mathrm{cm}^{3}\right)$

$$
\text { Retention }=\frac{\text { Moes }- \text { Moeo }}{\text { Moeo }} \times 100
$$

Equality;

Moes = Full dry weight of sample after impregnation (g)

Moeo $=$ Full dry weight of sample before impregnation (g)

$\mathrm{C}=$ Solution concentration (\%).

\section{Dry Layer Thicknesses Measurement}

In this study, dry layer thicknesses of water-based single and double component varnishes were determined by using PosiTector 200 device (Figure 3) according to ASTM D 6132 (2008) standard. With the film layers with various micron thicknesses, the probe of the calibrated device was printed on top of the gel (DeFelsko brand - Ultrasonic 
couplant) on wood material. Immediately after the device sent ultrasonic multiple signals, the values for micron thickness were automatically read on screen when scanning.

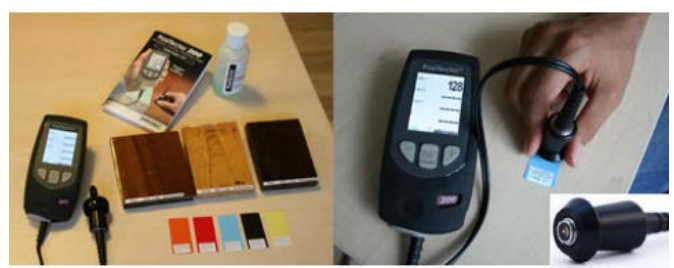

Figure 3. PosiTector 200 device (Ayata, 2014)

\section{Color Measurement}

The color changes of heat-treated and water-based varnishes (single and double component) applied samples were measured by using a Konica Minolta Chroma Meter CR-400 (Figure 4) (light source calibrated as D65, $10^{\circ}$ ).

Color parameters were taken using ten replicates of each sample and an average value was reported. The CIELAB system characterized by three parameters, $L^{*}, a^{*}$, and $b^{*}$ was used. The $L^{*}$ axis represents the lightness, plus $(+) a^{*}$ is the red, minus (-) $a^{*}$ for green, plus $(+) b^{*}$ for yellow, minus (-) $b^{*}$ for blue, and $L^{*}$ varies from 100 (white) to zero (black) (Zhang, Kamdem, \& Temiz, 2009). At the end of each exposure period, red color tone, yellow color tone and lightness difference values were determined according to ASTM-D 22443 (2007) standard. Total color differences $\left(\Delta E^{*}\right)$ were calculated through Equation 6.

$\Delta E^{*}=\left[\left(\Delta L^{*}\right)^{2}+\left(\Delta a^{*}\right)^{2}+\left(\Delta b^{*}\right)^{2}\right]^{1 / 2}(6)$

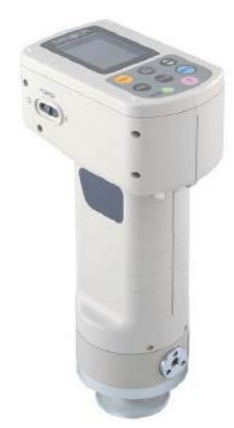

Figure 4. Konica Minolta Chroma Meter CR-400 (Ayata, 2014)

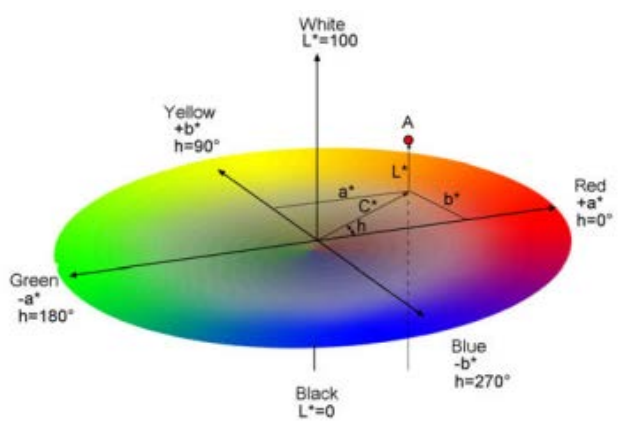

Figure 5. The three-dimensional CIEL $a^{*} b *$ colour space (Johansson, 2005).

\section{Statistical Evaluation}

Statistical analysis results were calculated. Total and single comparison tests, variance analysis and LSD (least significant difference) were determined for red color tone, yellow color tone, lightness and total color difference values. Statistical analysis results are tabulated.

\section{Results and Discussion}

While the highest solid content was found on water based single component varnish, the lowest solid content was obtained on impregnation colorless filler varnish (Table 3).

Table 3. Solid contents of varnishes

\begin{tabular}{lc}
\hline \multicolumn{1}{c}{ Varnish type } & Solid ratio (\%) \\
\hline Impregnation colorless filler varnish (FX 6150 UV) & 19.45 \\
\hline Water based single component varnish (FX 7680) & 43.26 \\
\hline Water based double component varnish (FX 0820 2K + AX & 37.78 \\
\hline 0115 Hardener) & \\
\hline
\end{tabular}


Table 4. Dry matter quantities and percent holding ratios of heat-treated wood species according to ThermoWood method

\begin{tabular}{|c|c|c|c|c|}
\hline Wood type & Heat treatment & Number of measurement & Dry Matter Quantity (kg/m³) & Hold Ratio (\%) \\
\hline \multirow{3}{*}{ Scots pine } & $190^{\circ} \mathrm{C}$ for 2 hours & 3 & 9.8089 & 10.34 \\
\hline & $212^{\circ} \mathrm{C}$ for 1 hour & 3 & 12.2580 & 11.86 \\
\hline & $212^{\circ} \mathrm{C}$ for 2 hours & 3 & 12.9515 & 14.05 \\
\hline \multirow{3}{*}{$\begin{array}{c}\text { Oriental } \\
\text { beech }\end{array}$} & $190^{\circ} \mathrm{C}$ for 2 hours & 3 & 6.2698 & 4.09 \\
\hline & $212^{\circ} \mathrm{C}$ for 1 hour & 3 & 6.8494 & 4.94 \\
\hline & $212^{\circ} \mathrm{C}$ for 2 hours & 3 & 7.5817 & 5.41 \\
\hline \multirow{3}{*}{ Oak } & $190^{\circ} \mathrm{C}$ for 2 hours & 3 & 6.5139 & 4.57 \\
\hline & $212^{\circ} \mathrm{C}$ for 1 hour & 3 & 6.6969 & 5.20 \\
\hline & $212^{\circ} \mathrm{C}$ for 2 hours & 3 & 6.8342 & 5.25 \\
\hline
\end{tabular}

Table 5. Layer thickness for varnishes

\begin{tabular}{cccccc}
\hline Heat Treatment & Varnish Type & Number of measurement & Scots pine $(\boldsymbol{\mu m})$ & Beech $(\boldsymbol{\mu m})$ & Oak $(\boldsymbol{\mu m})$ \\
\hline \multirow{2}{*}{$\mathbf{1 9 0}^{\circ} \mathbf{C}$ for $\mathbf{2}$ hours } & Single & $\mathbf{1 0}$ & 139.80 & 137.00 & 142.00 \\
\cline { 2 - 6 } & Double & $\mathbf{1 0}$ & 155.00 & 152.00 & 153.80 \\
\hline \multirow{2}{*}{$\mathbf{2 1 2}^{\circ} \mathbf{C}$ for $\mathbf{1}$ hour } & Single & $\mathbf{1 0}$ & 140.80 & 136.40 & 147.00 \\
\cline { 2 - 6 } & Double & $\mathbf{1 0}$ & 155.40 & 151.80 & 156.60 \\
\hline \multirow{2}{*}{$\mathbf{2 1 2}^{\circ} \mathbf{C}$ for $\mathbf{2}$ hours } & Single & $\mathbf{1 0}$ & 144.00 & 139.00 & 148.60 \\
\cline { 2 - 6 } & Double & $\mathbf{1 0}$ & 154.60 & 152.40 & 158.20 \\
\hline
\end{tabular}

Table 6. Results of variance analysis on effect of $\Delta a^{*}, \Delta b^{*}, \Delta L^{*}$ and $\Delta E^{*}$ values on wood type, heat treatment, varnish type and aging period

\begin{tabular}{|c|c|c|c|c|c|c|}
\hline Test & Variation Source & Degree of Freedom & Sum of squares & Average Square & F Value & $P \leq 0.05$ \\
\hline \multirow{17}{*}{ 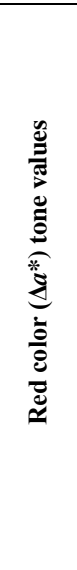 } & Wood type (A) & 2 & 4776.821 & 2388.411 & 6487.0816 & $0.0000 *$ \\
\hline & Heat treatment (B) & 2 & 494.405 & 247.203 & 671.4190 & $0.0000 *$ \\
\hline & Interaction (AB) & 4 & 487.208 & 121.802 & 330.8225 & $0.0000 *$ \\
\hline & Varnish type (C) & 1 & 0.139 & 0.139 & 0.3780 & $0.1048^{* *}$ \\
\hline & Interaction (AC) & 2 & 3.484 & 1.742 & 4.7308 & $0.0091^{*}$ \\
\hline & Interaction (BC) & 2 & 3.037 & 1.519 & 4.1246 & $0.0166^{*}$ \\
\hline & Interaction (ABC) & 4 & 70.604 & 17.651 & 47.9415 & $0.0000 *$ \\
\hline & Aging period (D) & 3 & 28.302 & 9.434 & 25.6237 & $0.0000 *$ \\
\hline & Interaction (AD) & 6 & 152.352 & 25.392 & 68.9665 & $0.0000 *$ \\
\hline & Interaction (BD) & 6 & 57.782 & 9.630 & 26.1567 & $0.0000 *$ \\
\hline & Interaction (ABD) & 12 & 34.190 & 2.849 & 7.7385 & $0.0000^{*}$ \\
\hline & Interaction (CD) & 3 & 0.486 & 0.162 & 0.4401 & $0.1044^{* *}$ \\
\hline & Interaction (ACD) & 6 & 8.319 & 1.387 & 3.7660 & $0.0011^{*}$ \\
\hline & Interaction (BCD) & 6 & 9.929 & 1.655 & 4.4946 & $0.0002 *$ \\
\hline & $\begin{array}{l}\text { Interaction } \\
\text { (ABCD) }\end{array}$ & 12 & 9.961 & 0.830 & 2.2546 & $0.0085^{*}$ \\
\hline & Error & 648 & 238.580 & 0.368 & & \\
\hline & Total & 719 & 6375.602 & & & \\
\hline \multirow{17}{*}{ 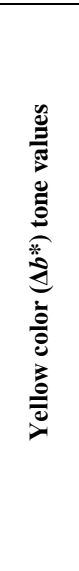 } & Wood type (A) & 2 & 33911.133 & 16955.567 & 13775.0690 & $0.0000^{*}$ \\
\hline & Heat treatment (B) & 2 & 7142.748 & 3571.374 & 2901.4613 & $0.0000 *$ \\
\hline & Interaction (AB) & 4 & 1763.491 & 440.873 & 358.1746 & $0.0000 *$ \\
\hline & Varnish type (C) & 1 & 5.486 & 5.486 & 4.4572 & $0.0351 *$ \\
\hline & Interaction (AC) & 2 & 5.726 & 2.863 & 2.3259 & $0.0985^{* *}$ \\
\hline & Interaction (BC) & 2 & 64.360 & 32.180 & 26.1436 & $0.0000^{*}$ \\
\hline & Interaction (ABC) & 4 & 328.411 & 82.103 & 66.7021 & $0.0000 *$ \\
\hline & Aging period (D) & 3 & 828.258 & 276.086 & 224.2982 & $0.0000^{*}$ \\
\hline & Interaction (AD) & 6 & 111.283 & 18.547 & 15.0682 & $0.0000 *$ \\
\hline & Interaction (BD) & 6 & 127.020 & 21.170 & 17.1990 & $0.0000^{*}$ \\
\hline & Interaction (ABD) & 12 & 185.548 & 15.462 & 12.5619 & $0.0000^{*}$ \\
\hline & Interaction (CD) & 3 & 38.774 & 12.925 & 10.5001 & $0.0000 *$ \\
\hline & Interaction (ACD) & 6 & 22.061 & 3.677 & 2.9872 & $0.0069 *$ \\
\hline & Interaction (BCD) & 6 & 52.437 & 8.740 & 7.1002 & $0.0000^{*}$ \\
\hline & $\begin{array}{l}\text { Interaction } \\
\text { (ABCD) }\end{array}$ & 12 & 41.327 & 3.444 & 2.7979 & $0.0010^{*}$ \\
\hline & Error & 648 & 797.615 & 1.231 & & \\
\hline & Total & 719 & 45425.678 & & & \\
\hline \multirow{7}{*}{ 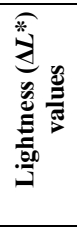 } & Wood type (A) & 2 & 35297.321 & 17648.660 & 33728.2846 & $0.0000 *$ \\
\hline & Heat treatment (B) & 2 & 14216.579 & 7108.290 & 13584.6242 & $0.0000 *$ \\
\hline & Interaction (AB) & 4 & 3072.084 & 768.021 & 1467.7620 & $0.0000 *$ \\
\hline & Varnish type (C) & 1 & 81.925 & 81.925 & 156.5661 & $0.0000 *$ \\
\hline & Interaction (AC) & 2 & 14.585 & 7.292 & 13.9364 & $0.0000 *$ \\
\hline & Interaction (BC) & 2 & 28.514 & 14.257 & 27.2467 & $0.0000 *$ \\
\hline & Interaction (ABC) & 4 & 248.450 & 62.113 & 118.7031 & $0.0000^{*}$ \\
\hline
\end{tabular}


Table 6 (continued)

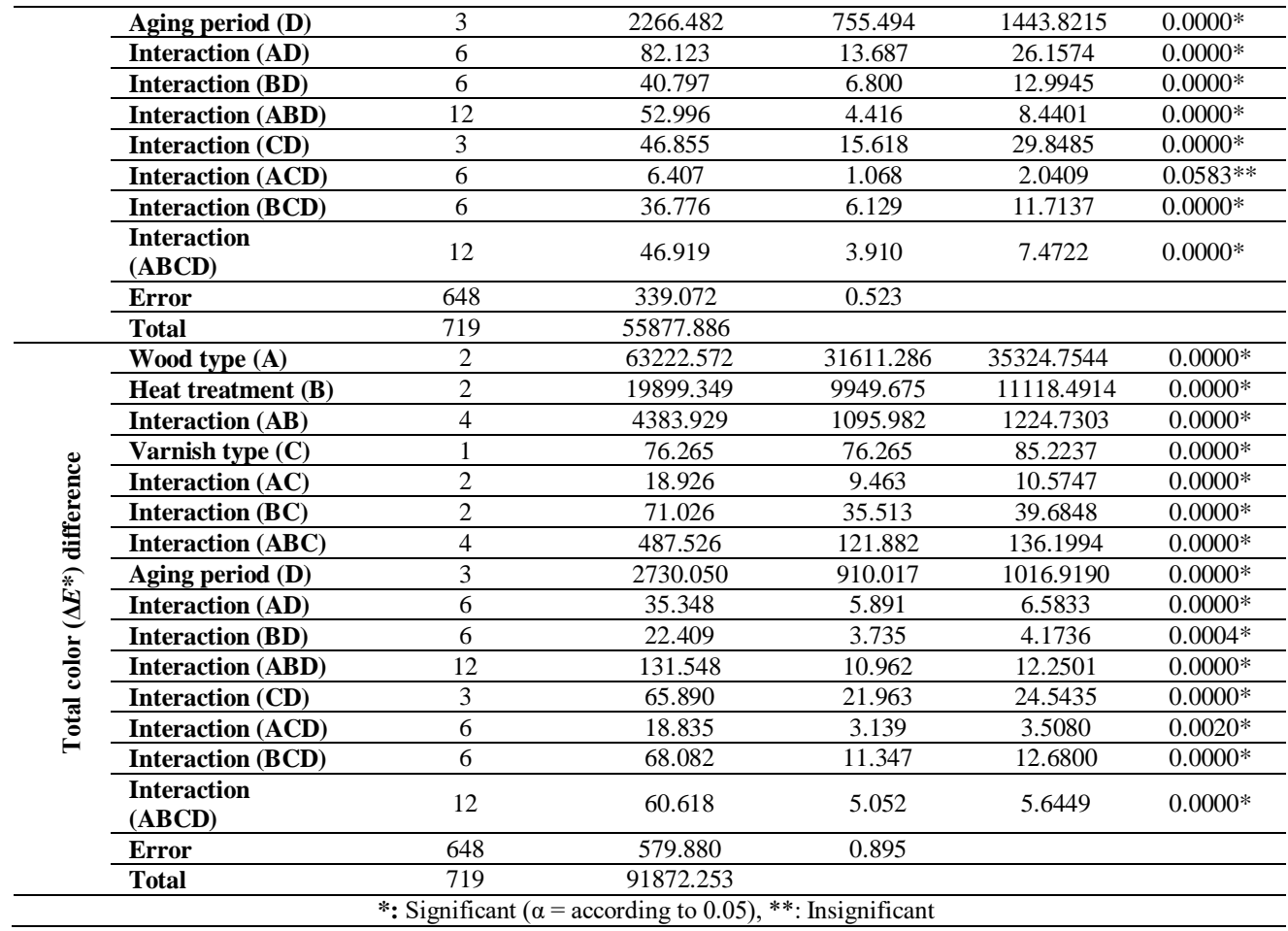

The amounts of net dry matter and retention increased when increasing heat treatment time and temperature (Table 4).

While the highest dry matter content and retention rate were obtained on Scots pine wood heat-treated at $212^{\circ} \mathrm{C}$ for 2 hours, the lowest values were determined on beech heat-treated at $190^{\circ} \mathrm{C}$ for 2 hours.
The measurement results of dry film thicknesses of water-based single and double component varnishes are given in Table 5. The highest layer thickness was found oak heat-treated at $212^{\circ} \mathrm{C}$ for 2 hours and water-based double component varnished samples. But the lowest layer thickness was obtained beech heat-treated at $212^{\circ} \mathrm{C}$ for 1 hour and water-based single component varnished (Table 5). 
Table 7. Single comparison results of wood type - heat treatment - varnish type - aging period for $\Delta a^{*}, \Delta b^{*}, \Delta L^{*}$ and $\Delta E^{*}$ values

\begin{tabular}{|c|c|c|c|c|c|c|c|c|c|}
\hline & \multirow[t]{2}{*}{ Factor } & \multicolumn{3}{|c|}{$\begin{array}{c}\text { Red color }\left(\Delta a^{*}\right) \text { tone } \\
\text { value }\end{array}$} & & \multirow[t]{2}{*}{ Factor } & \multicolumn{3}{|c|}{$\begin{array}{c}\text { Yellow color }\left(\Delta b^{*}\right) \text { tone } \\
\text { value }\end{array}$} \\
\hline & & $\mathbf{X}$ & $H G$ & LSD \pm & & & $\mathbf{X}$ & $H G$ & LSD \pm \\
\hline \multirow{3}{*}{$\begin{array}{l}\text { Wood } \\
\text { type }\end{array}$} & Scots pine & 12.51 & $\mathrm{~A}^{*}$ & \multirow{3}{*}{0.1087} & \multirow{3}{*}{$\begin{array}{l}\text { Wood } \\
\text { type }\end{array}$} & Scots pine & 24.46 & $\mathrm{~A}^{*}$ & \multirow{3}{*}{0.1989} \\
\hline & Beech & 9.26 & $\mathrm{~B}$ & & & Beech & 13.68 & $\mathrm{~B}$ & \\
\hline & Oak & 6.20 & $\mathrm{C}$ & & & Oak & 7.90 & $\mathrm{C}$ & \\
\hline \multirow{3}{*}{$\begin{array}{c}\text { Heat } \\
\text { Treat- } \\
\text { ment }\end{array}$} & $\begin{array}{c}190^{\circ} \mathrm{C} \text { for } 2 \\
\text { hours }\end{array}$ & 10.38 & $A^{*}$ & \multirow{3}{*}{0.1087} & \multirow{3}{*}{$\begin{array}{c}\text { Heat } \\
\text { Treat- } \\
\text { ment }\end{array}$} & $\begin{array}{c}190^{\circ} \mathrm{C} \text { for } 2 \\
\text { hours }\end{array}$ & 19.62 & $A^{*}$ & \multirow{3}{*}{0.1989} \\
\hline & $\begin{array}{c}212^{\circ} \mathrm{C} \text { for } 1 \\
\text { hour }\end{array}$ & 9.25 & B & & & $212^{\circ} \mathrm{C}$ for 1 hour & 14.31 & B & \\
\hline & $\begin{array}{c}212^{\circ} \mathrm{C} \text { for } 2 \\
\text { hours }\end{array}$ & 8.35 & $\mathrm{C}$ & & & $\begin{array}{c}212^{\circ} \mathrm{C} \text { for } 2 \\
\text { hours }\end{array}$ & 12.11 & $\mathrm{C}$ & \\
\hline \multirow{2}{*}{$\begin{array}{c}\text { Varnish } \\
\text { type }\end{array}$} & Single & 26.27 & $\mathrm{~B}$ & \multirow{2}{*}{0.1627} & \multirow{2}{*}{$\begin{array}{c}\text { Varnish } \\
\text { type }\end{array}$} & Single & 15.26 & $\mathrm{~B}$ & \multirow{2}{*}{0.1624} \\
\hline & Double & 26.64 & $\mathrm{~A}^{*}$ & & & Double & 15.44 & $\mathrm{~A}^{*}$ & \\
\hline \multirow{4}{*}{$\begin{array}{l}\text { Aging } \\
\text { period }\end{array}$} & Control & 9.19 & $\mathrm{C}$ & \multirow{4}{*}{0.1256} & \multirow{4}{*}{$\begin{array}{l}\text { Aging } \\
\text { period }\end{array}$} & Control & 13.92 & $\mathrm{C}$ & \multirow{4}{*}{0.2297} \\
\hline & 144 hours & 9.08 & $\mathrm{C}$ & & & 144 hours & 14.70 & $\mathrm{~B}$ & \\
\hline & 288 hours & 9.58 & A* & & & 288 hours & 16.36 & $\mathrm{~A}$ & \\
\hline & 432 hours & 9.44 & $\mathrm{~B}$ & & & 432 hours & 16.41 & A* & \\
\hline \multirow{2}{*}{\multicolumn{2}{|c|}{ Factor }} & \multicolumn{3}{|c|}{ Lightness $\left(\Delta L^{*}\right)$ value } & \multirow{2}{*}{\multicolumn{2}{|c|}{ Factor }} & \multicolumn{3}{|c|}{ Total color $\left(\Delta E^{*}\right)$ difference } \\
\hline & & $\mathbf{X}$ & HG & LSD \pm & & & $\mathbf{X}$ & HG & LSD \pm \\
\hline \multirow{3}{*}{$\begin{array}{l}\text { Wood } \\
\text { type }\end{array}$} & Scots pine & 47.53 & $\mathrm{~A}^{*}$ & \multirow{3}{*}{0.1296} & \multirow{3}{*}{$\begin{array}{l}\text { Wood } \\
\text { type }\end{array}$} & Scots pine & 54.95 & A* & \multirow{3}{*}{0.1696} \\
\hline & Beech & 37.31 & $\mathrm{~B}$ & & & Beech & 40.93 & $\mathrm{~B}$ & \\
\hline & Oak & 30.49 & $\mathrm{C}$ & & & Oak & 32.20 & $\mathrm{C}$ & \\
\hline \multirow{3}{*}{$\begin{array}{c}\text { Heat } \\
\text { Treat- } \\
\text { ment }\end{array}$} & $\begin{array}{c}190^{\circ} \mathrm{C} \text { for } 2 \\
\text { hours }\end{array}$ & 44.53 & $A^{*}$ & \multirow{3}{*}{0.1296} & & $\begin{array}{c}190^{\circ} \mathrm{C} \text { for } 2 \\
\text { hours }\end{array}$ & 49.87 & $A^{*}$ & \\
\hline & $\begin{array}{l}212^{\circ} \mathrm{C} \text { for } 1 \\
\text { hour }\end{array}$ & 36.74 & B & & Treat- & $212^{\circ} \mathrm{C}$ for 1 hour & 40.77 & B & 0.1696 \\
\hline & $\begin{array}{c}212^{\circ} \mathrm{C} \text { for } 2 \\
\text { hours }\end{array}$ & 34.06 & $\mathrm{C}$ & & & $\begin{array}{c}212^{\circ} \mathrm{C} \text { for } 2 \\
\text { hours }\end{array}$ & 37.43 & $\mathrm{C}$ & \\
\hline Varnish & Single & 38.11 & $\mathrm{~B}$ & 0.1058 & Varnish & Single & 42.37 & $\mathrm{~B}$ & 0.1385 \\
\hline type & Double & 38.78 & $\mathrm{~A}^{*}$ & 0.1058 & type & Double & 43.02 & $\mathrm{~A}^{*}$ & 0.1385 \\
\hline & Control & 35.68 & $\mathrm{D}$ & & & Control & 39.77 & $\mathrm{D}$ & \\
\hline Aging & 144 hours & 38.12 & $\mathrm{C}$ & & Aging & 144 hours & 42.09 & $\mathrm{C}$ & 01958 \\
\hline period & 288 hours & 39.75 & $\mathrm{~B}$ & 0.1497 & period & 288 hours & 44.29 & $\mathrm{~B}$ & 0.1958 \\
\hline & 432 hours & 40.22 & $\mathrm{~A}^{*}$ & & & 432 hours & 44.62 & $A^{*}$ & \\
\hline
\end{tabular}

Table 6 shows the results of variance analysis for $\Delta L^{*}, \Delta a^{*}, \Delta b^{*}$ and $\Delta E^{*}$. According to varnish type (C) and interaction $(\mathrm{CD})$ for $\Delta a^{*}$, interaction (AC) for $\Delta b^{*}$ and interaction (ACD) for $\Delta L^{*}$ were not significant (Table 6). In addition, all other factors and interactions were significant at $\Delta L^{*}, \Delta a^{*}, \Delta b^{*}$ and $\Delta E^{*}$ values.

Single comparison results of wood type - heat treatment - varnish type aging period for $\Delta a^{*}, \Delta b^{*}, \Delta L^{*}$ and $\Delta E^{*}$ values are given in Table 7 . According to Table 7, for wood type, Scots pine wood was determined highest when Oak wood was found lowest $\Delta a^{*}, \Delta b^{*}, \Delta L^{*}$ and
$\Delta E^{*}$ values. At heat treatment level, $190^{\circ} \mathrm{C}$ for 2 hours were obtained highest while $212^{\circ} \mathrm{C}$ for 2 hours were found lowest $\Delta b^{*}, \Delta a^{*}, \Delta L^{*}$ and $\Delta E^{*}$ values. For varnish type, double component varnish was determined highest when single component varnish was found lowest $\Delta b^{*}, \Delta L^{*}$ and $\Delta E^{*}$ values. At aging period level, 432 hours were given highest while control (unaged) period was given lowest $\Delta L^{*}, \Delta b^{*}$ and $\Delta E^{*}$ values. While highest $\Delta a^{*}$ value was determined at 288 hours, lowest $\Delta a^{*}$ value was found at 144 hours for aging period level. 
Table 8. Total comparison results of wood type - heat treatment - varnish type - aging period for $\Delta a^{*}, \Delta b^{*}, \Delta L^{*}$ and $\Delta E^{*}$ values

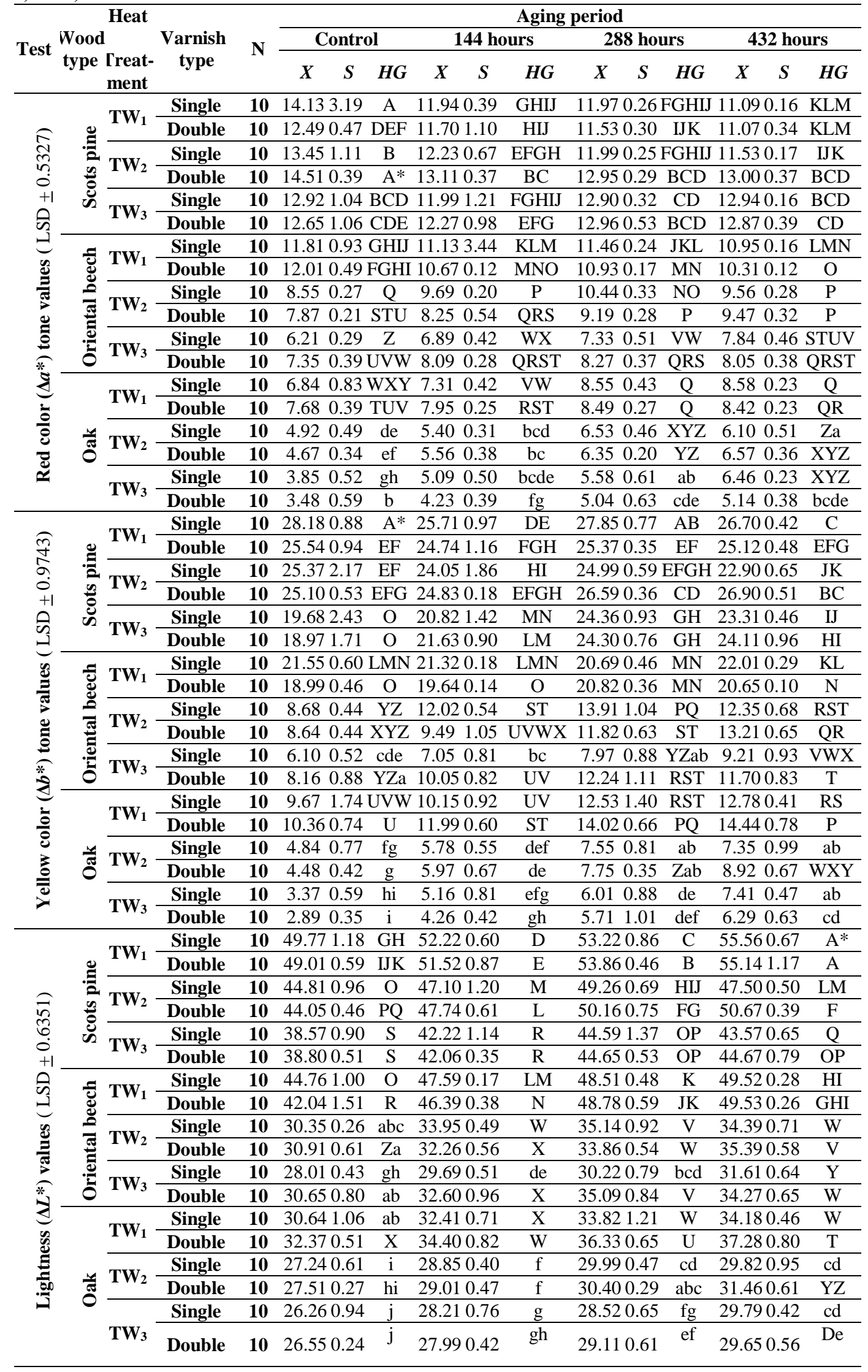


Table 8. (continued)

\begin{tabular}{|c|c|c|c|c|c|c|c|c|c|c|c|c|}
\hline \multirow{6}{*}{ 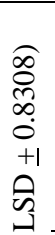 } & \multirow{6}{*}{ 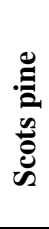 } & \multirow{2}{*}{$\mathbf{T W}_{\mathbf{1}}$} & Single & 10 & 58.991 .79 & $\mathrm{DE}$ & 59.420 .90 & $\mathrm{D}$ & 61.250 .85 & $\mathrm{BC}$ & 62.640 .62 & A* \\
\hline & & & Double & 10 & 56.660 .92 & $\mathrm{~F}$ & 58.411 .13 & $\mathrm{E}$ & 60.650 .43 & $\mathrm{C}$ & 61.601 .04 & $\mathrm{~B}$ \\
\hline & & \multirow{2}{*}{$\mathrm{TW}_{2}$} & Single & 10 & 53.251 .71 & IJ & 54.291 .96 & $\mathrm{H}$ & 56.530 .73 & $\mathrm{~F}$ & 53.980 .70 & $\mathrm{HI}$ \\
\hline & & & Double & 10 & 52.740 .65 & JK & 55.390 .47 & $G$ & 58.230 .69 & $\mathrm{E}$ & 58.820 .53 & $\mathrm{DE}$ \\
\hline & & \multirow{2}{*}{$\mathbf{T W}_{3}$} & Single & 10 & 45.021 .66 & $\mathrm{O}$ & 48.611 .16 & $M$ & 52.421 .54 & K & 51.090 .74 & $\mathrm{~L}$ \\
\hline & & & Double & 10 & 45.041 .30 & $\mathrm{O}$ & 48.930 .54 & $M$ & 52.470 .80 & JK & 52.371 .05 & K \\
\hline \multirow{7}{*}{ 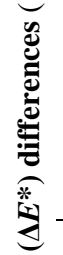 } & \multirow{6}{*}{ 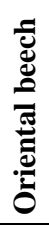 } & \multirow{2}{*}{$\mathbf{T W}_{\mathbf{1}}$} & Single & 10 & 51.071 .09 & $\mathrm{~L}$ & 53.280 .22 & $\mathrm{IJ}$ & 54.770 .59 & $\mathrm{GH}$ & 55.290 .32 & $\mathrm{G}$ \\
\hline & & & Double & 10 & 47.671 .46 & $\mathrm{~N}$ & 51.490 .37 & $\mathrm{~L}$ & 54.130 .66 & $\mathrm{H}$ & 54.640 .25 & $\mathrm{GH}$ \\
\hline & & \multirow{2}{*}{$\mathrm{TW}_{2}$} & Single & 10 & 32.710 .41 & WX & 37.300 .56 & ST & 39.211 .27 & QR & 37.780 .92 & ST \\
\hline & & & Double & 10 & 33.070 .69 & VW & 34.570 .95 & $\mathrm{U}$ & 37.020 .73 & $\mathrm{~T}$ & 38.940 .83 & $\mathrm{R}$ \\
\hline & & \multirow{2}{*}{$\mathrm{TW}_{3}$} & Single & 10 & 29.340 .51 & bcd & 31.290 .76 & $\mathrm{YZ}$ & 32.101 .08 & $\mathrm{XY}$ & 33.740 .85 & $\mathrm{~V}$ \\
\hline & & & Double & 10 & 32.571 .01 & WX & 35.071 .10 & $\mathrm{U}$ & 38.081 .18 & $\mathrm{~S}$ & 37.130 .87 & $\mathrm{~T}$ \\
\hline & \multirow{6}{*}{ है } & \multirow{2}{*}{$\mathbf{T W}_{1}$} & Single & 10 & 32.811 .60 & WX & 34.750 .91 & $\mathrm{U}$ & 37.081 .67 & $\mathrm{~T}$ & 37.490 .58 & ST \\
\hline \multirow{5}{*}{$\frac{\frac{0}{0}}{\frac{0}{0}}$} & & & Double & 10 & 34.850 .72 & $\mathrm{U}$ & 37.290 .97 & ST & 39.850 .87 & $\mathrm{Q}$ & 40.851 .04 & $\mathrm{P}$ \\
\hline & & \multirow{2}{*}{$\mathbf{T W}_{2}$} & Single & 10 & 28.110 .73 & e & 29.920 .54 & $a b$ & 31.610 .71 & $\mathrm{Y}$ & 31.321 .23 & $\mathrm{YZ}$ \\
\hline & & & Double & 10 & 28.270 .34 & $\mathrm{e}$ & 30.140 .65 & $\mathrm{ab}$ & 32.010 .38 & $\mathrm{XY}$ & 33.360 .82 & VW \\
\hline & & \multirow{2}{*}{$\mathbf{T W}_{3}$} & Single & 10 & 26.761 .06 & $\mathrm{f}$ & 28.930 .96 & cde & 29.690 .90 & bc & 31.380 .55 & $\mathrm{YZ}$ \\
\hline & & & Double & 10 & 26.940 .29 & $\mathrm{f}$ & 28.530 .50 & de & 30.100 .88 & $\mathrm{ab}$ & 30.750 .72 & $\mathrm{Za}$ \\
\hline & & & & & & & alue, & & & & leasureme & \\
\hline
\end{tabular}

Total comparison results of wood type - heat treatment - varnish type - aging period for $\Delta a^{*}, \Delta b^{*}, \Delta L^{*}$ and $\Delta E^{*}$ values are given in Table 8. According to Table 8 , highest $\Delta a^{*}$ was found in control (unaged) samples on scots pine specimens varnished with double components varnish and heat-treated at $212^{\circ} \mathrm{C}$ for 1 hour when highest $\Delta b^{*}$ was found in control (unaged) period of scots pine samples varnished with single component varnish and heat-treated at $190^{\circ} \mathrm{C}$ for 2 hours. Highest $\Delta L^{*}$ and $\Delta E^{*}$ values were found at the end of 432 hours weathering period on scots pine specimens varnished with single component varnish and heat-treated at $190^{\circ} \mathrm{C}$ for 2 hours (Table 8). When lowest $\Delta b^{*}$ and $\Delta a^{*}$ values were determined in control period of oak specimens varnished with double component varnish and heat-treated at $212^{\circ} \mathrm{C}$ for 2 hours, lowest $\Delta L^{*}$ and $\Delta E^{*}$ were found in control specimens of oak specimens varnished with single component varnish and heat-treated at $212^{\circ} \mathrm{C}$ for 2 hours for wood type - heat treatment - varnish type - aging period (Table 8).

\section{Conclusion}

In this study, effect of UV weathering on color values of Scotch pine, Oak and Oriental beech wood species heat-treated according to ThermoWood method $\left(190^{\circ} \mathrm{C}\right.$ for 2 hours, $212^{\circ} \mathrm{C}$ for 1 hour and 2 hours) and water-based varnishes (single and double component) applied were investigated. At the aging period level, for the red color value, 288 hour UV aging samples were given highest, 144 hours UV aging samples were given lowest. A statistical difference was observed between control and 144 hour aging samples and between 288 and 432 hour aging samples. This difference is thought to be caused by the effect of UVrays on the varnish layer during long aging periods. In addition, the binder resins in the single component varnish formulation may have interfered with the wood extracts. At the aging period level, the yellow color value was highest in the 432 hour UV aging samples and it was lowest in the control samples. Accordingly, it can be said that the aging process was an effect of increasing the 
yellow color value. At the aging period level, the highest lightness value was determined at 432 hours UV aging samples, lowest lightness was obtained in the lowest control samples. When highest was obtained on pine wood, lowest was found on oak wood at wood species level for yellow color tone, red color tone and lightness values. While highest was determined on heat-treated at $190^{\circ} \mathrm{C}$ for 2 hours samples, lowest was found on heattreated at $212^{\circ} \mathrm{C}$ for 2 hours samples at heat treatment level for yellow color tone, red color tone and lightness values. In addition, total color difference value was increased by increasing the aging period. The Feist (1984) study reported that the high energy of sunlight ultraviolet (UV) wave lengths caused deterioration of the varnish and paint layers. In a study done by Payne (1965), it has been reported in the literature that the total color difference values are high in accelerated aging samples.

\section{Acknowledgements}

This study was produced from a doctoral thesis titled "Determination of the resistance of water based layers on some heat-treated (ThermoWood) wood species against accelerated UV aging" and supported by Duzce University BAP2012.02.HD.078 Scientific Research Project.

\section{Referances}

Akkus, M. (2012). The effects of bleaching process on some wood species modified with thermal treatment, Duzce University, Institute of Science and Technology, Department of Furniture and Decoration Education Master of Science Thesis, Duzce, Turkey.

Anonymous, (2003). ThermoWood Handbook, Finnish ThermoWood Association, Helsinki- Finland.

ASTM D 1644-01, (2006). Standard Test Methods for Nonvolatile Content of Varnishes. American Society for Testing and Materials, West Conshohocken, Pennsylvania, USA.

ASTM D 2244-3, (2007). Standard practice for calculation or color tolerances and color differences from instrumentally measured color coordinates, ASTM Standards, A.B.D., 1-13.

ASTM D 6132, (2008). Standard test method for nondestructive measurement of dry film thickness of applied organic coatings using an ultrasonic gage.

ASTM G 154-06, (2006). Standard practice for operating fluorescent light apparatus for UV exposure of nonmetallic materials, ASTM, USA, 2-8.

Ayata, U. (2014). Determination of the resistance of water based layers on some heat treated (ThermoWood) wood species against accelerated UV aging, Duzce University, Graduate School of Natural and Applied Sciences, Department of Forest Industry Engineering, Doctoral Thesis, Duzce, Turkey.

Bozkurt, Y., Goker, Y., \& Erdin, N. (1993). Emprenye Tekniği, İ.Ü. Orman Fakültesi Yayınları, Istanbul, 3779(425), 125 ve 429.

Cakicier, N. (2007). Changes due to weathering of surface finishing layers of wood, İstanbul University, Institute of Science and Technology, PhD Thesis, Istanbul, Turkey.

Feist, W. C. (1984). Painting and finishing wood for use outdoor, Forest Product Laboratory Report, 401-454.

Gonzalez-Pena, M. M., \& Hale Michael, D. C. (2009). Colour in thermally modified wood of beech, Norway spruce and Scots pine. Part 1: Colour Evolution and Colour Changes, Holzforschung, 63, 385-393.

Hillis, W. E. (1975). The role of wood characteristics in high temperature drying, Journal Industrial Wood Science, 7(2), 60-67.

Johansson, D., (2005). Strenght and colour response of solid wood to heat treatment, Graduate Thesis, Luleå University of Technology, Department of Skelleftea Campus, Division of Wood Technology, Sweden, 93(5), 1402-1757.

Kazan, B., (2009). Su bazlı vernik uygulanmış yüzey üzerindeki ısıl işlemin etkileri, Dumlupınar Üniversitesi, Fen 
Bilimleri Enstitüsü, Mobilya ve Dekorasyon Egitimi, Yüksek Lisans Tezi, Dumlupinar.

Korkut, S., \& Kocaefe, D. (2009). Effect of heat treatment on wood properties, Duzce University Journal of Forestry, 5(2), 1134.

Kurtoğlu, A. (2000). Ağaç Malzeme Yüzey İşlemleri, Genel Bilgiler, Cilt I, İ.Ü. Orman Fak. Orman End. Müh. Böl., İstanbul.

Payne, H. F. (1965). Organic coating technology, volume I, printed in New York U.S.A. Fourth Printing.

Soğutlu, C., \& Sonmez A. (2006). The effect of UV lights on color changes on some local wood processed with differential preservatives, J. Fac. Eng. Arch. Gazi Univ., 21(1), 151-159.

TS 642 ISO 554, (1997). Standard atmospheres for conditioning and/or testing; Specifications, Turkish Standards Institution, Ankara.

TS 5723, (1988). Wood preservation Penetration test, Turkish Standards Institution, Ankara.

Zhang, J., Kamdem, D. P., \& Temiz, A. (2009). Weathering of copper-amine treated wood, Appl. Surf. Sci., 256(3), 842-846 